\title{
SYMPATHETIC SKIN RESPONSE - CORRELATION WITH THE SEVERITY OF CARDIOVASCULAR AUTONOMIC DYSFUNCTION IN PATIENTS WITH ISCHEMIC CEREBRAL STROKE
}

\author{
Darina Georgieva-Hristova \\ Department of Neurology and Neurosciences, Second Clinic of Neurology, \\ Medical University of Varna
}

\begin{abstract}
The sympathetic skin response (SSR) is a polysynaptic somatosympathetic multiaxonal reflex with a spinal, bulbar and suprabulbar component. From a pathogenetic point of view it is considered that the SSR changes are a result of an interruption of the efferent sympathetic pathways. The study of the correlation between the SSR parameters and the severity of the cardiovascular autonomic dysfunction in patients with acute ischemic stroke is a matter of interest. The data from our research showed that the patients with severe autonomic dysfunction had a significantly more expressed SSR parameter disorders than the deviations in patients with milder autonomic dysfunctions.
\end{abstract}

Keywords: sympathetic skin response, stroke, cardiovascular autonomic dysfunction

\section{INTRODUCTION}

The disturbances of the sympathetic flow are well-known consequences after a hemispheric or a brain stem damage (1). Although the exact pathogenetic mechanism is not clear, they are often associated with an increased activity of the sympathetic nervous system.

SSR is a polysynaptic somatosympathetic multiaxonal reflex with a spinal, bulbar and suprabulbar component (2). It is generated by various stimuli, which illustrates the complexity of the afferent input information of the reflex (3) but it has a common

\footnotetext{
Address for correspondence:

Darina Georgieva

Department of Neurology,

Medical University of Varna

55 Marin Drinov Str.

9002 Varna

e-mail:darina_georgieva@abv.bg
}

Received: November 14, 2016

Accepted: December 12, 2016 efferent pathway in the spinal cord. The final efferent pathway includes pre- and postganglionic sympathetic sudomotor fibers and effector organs - the sweat glands (4).

The sympathetic sudomotor dysfunction during the acute stage of an ischemic stroke can be related to a weakened or missing excitatory effect of the cortical structures on the sympathetic division of the autonomic nervous system which has been partially restored during the chronic stage due to cortical compensation and neurological recovery (5). From a pathogenetic point of view, it is considered that the SSR changes are a result of an interruption of the efferent sympathetic pathways the severity of which depends on the character of the brain damage as well as on the cerebral edema (6).

The basic SSR parameters are latency, amplitude, and duration. They are affected by environmental factors, body temperature, position of the body, physical activity, various stimulations, emo- 
tional state, sudden stress from stimulation, adjusting to stimulation, and circadian rhythms (2).

Prolonged latency or asynchronous response are assumed to be abnormality criteria when they correlate with disturbances in other tests of the autonomic function (7).

JT. Koperlainen et al. clarify that the SSR sensitivity is dependent on the degree of reflex activity presented in Table 1. Among the patients, the largest groups are the one of the patients with a stroke in the territory of the right middle cerebral artery (RMCA) without involvement of the insular cortex (IC) $-22.9 \%(n=16)$ and the one of patients with a stroke in the territory of the left middle cerebral artery (LMCA) without involvement of the IC $-21.4 \%$ $(\mathrm{n}=15)$. The lowest number was in the group of patients with ischemic stroke in RMCA with IC -15.7\%

Table 1. Distribution of the studied groups

\begin{tabular}{l|c|c|c|c|c|}
\hline \multirow{2}{*}{$\begin{array}{l}\text { Distribution by } \\
\text { groups }\end{array}$} & Controls & RMCA with IC & RMCA w/o IC & LMCA with IC & LMCA w/o IC \\
\cline { 2 - 6 } & $28.6 \%$ & $15.7 \%$ & $22.9 \%$ & $11.4 \%$ & $21.4 \%$ \\
$(\mathrm{n}=20)$ & $(\mathrm{n}=11)$ & $(\mathrm{n}=16)$ & $(\mathrm{n}=8)$ & $(\mathrm{n}=15)$ \\
\hline
\end{tabular}

suppression of the sympathetic nervous system.

The study of the correlation between the SSR parameters and the severity of the cardiovascular autonomic dysfunction in patients with acute ischemic stroke is a matter of interest. As far as we know, there has been only one clinical research, which has studied this correlation and the results are summarized and presented only as an abstract (8). The authors proved the correlation dependence between the degree of severity of the autonomic dysfunctions, evaluated through the Ewing's battery, and the SSR changes and confirmed the clinical advantage of this neurophysiological method for autonomic function evaluation. They also reported that the results from the autonomic tests were not influenced by age, sex, duration of the disease, etiology, and localization of the stroke.

\section{AIM}

The aim of this paper is to study the correlation between the SSR parameter changes and the severity of the cardiovascular autonomic dysfunction in patients with acute hemispheric ischemic cerebral stroke (ICS).

\section{MATERIALS AND METHODS}

We studied 50 patients with acute hemispheric ischemic cerebral stroke and 20 clinically healthy volunteers. The studied subjects were selected by specific inclusion and exclusion criteria.

For the purposes of our research the patients were divided into groups based on the localization of the stroke. The results of the 70 individuals are $(\mathrm{n}=11)$ and the one of patients with stroke in LMCA with IC $-11.4 \%(n=8)$.

The research was performed with a two-channel electromyograph (Neuron-Spectrum-5) (Fig. 1 and 2) using the following technical parameters: electrical stimulation of a sensory nerve (n. medianus, n.tibialis) with a duration of $0.2-0.5 \mathrm{~ms}$ and ap-

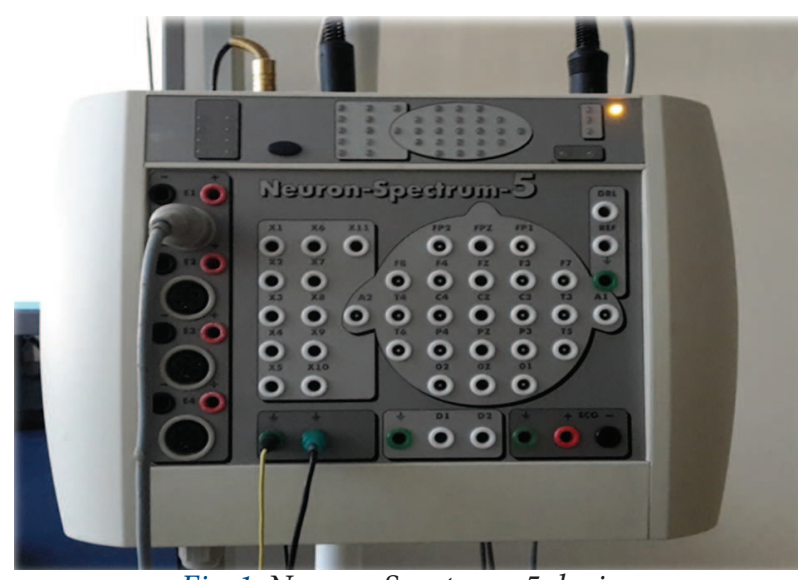

Fig. 1. Neuron-Spectrum-5 device

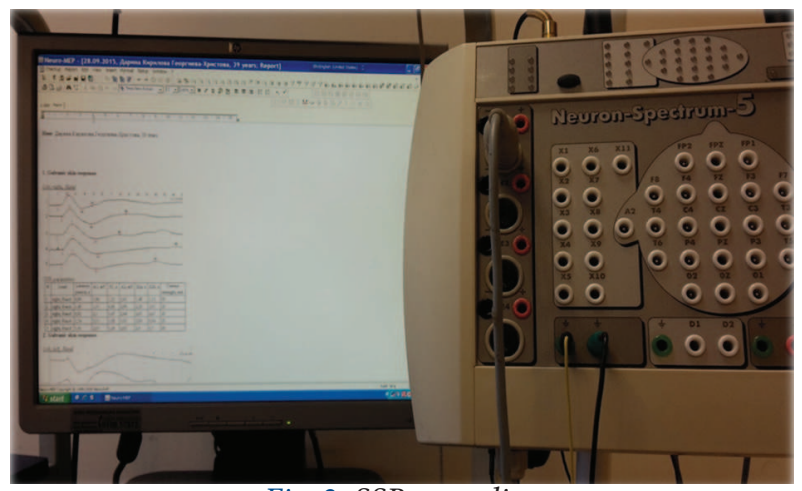

Fig. 2. SSR recording 
plication of a gradually increasing electrical stimulation of 20-40 mA, at intervals of 60 to $120 \mathrm{~ms}$. The length of the recording was 5 to 10 seconds. The stimulations were applied at irregular intervals of time with an approximate interval of one minute between stimuli to avoid adjusting.

The lead was done with standard surface electrodes on the hand and the leg using electrolyte gel. When leading the hands, the active electrode was positioned on the palm and the indifferent one on the back of the hand. When leading the legs, the active electrode was placed on the sole of the foot and the indifferent one - on the back of the sole.

We analyzed the latency time and the amplitude of the highest of the five consecutively recorded potentials registered on the hands and the legs, left and right, respectively, during ipsilateral stimulation.

In order to establish the correlation level between the latency times and the severity of autonomic dysfunctions, correlation and regression analyses were performed.

\section{RESULTS}

By the classification of Ewing, the results from each test were defined as: normal, border, and abnormal and the autonomic dysfunctions were classified as: normal, mild, marked, severe, and atypical. For the purposes of our research, we divided the patients into two groups: group 1 - with normal or mild dysfunctions of the cardiovascular autonomic function and group 2 - with marked, severe or atypical dysfunctions.

The results from the correlation and regression analysis showed a strong correlation between the latency and the severity of the autonomic dysfunction (AD) $(r=0.58 ; \mathrm{p}=0.001)$. Respectively, the predictive latency value related to the severity of the $\mathrm{AD}$ was $32 \%(\mathrm{R} 2=0.321 ; \mathrm{p}=0.001)$. The strong correlation and regression connections of the indicators are shown on Fig. 3.

As with latency, the amplitude also has a statistically significant correlation with the $\mathrm{AD}$ severity $(\mathrm{r}=0.615 ; \mathrm{p}=0.001)$. The predictive value of the amplitude is 35\% in regard to the development of cardiovascular autonomic dysfunctions $(\mathrm{R} 2=0.357 ; \mathrm{p}=$ $0.001)$. The strong correlation and regression connections of the indicators are shown on Fig. 4.

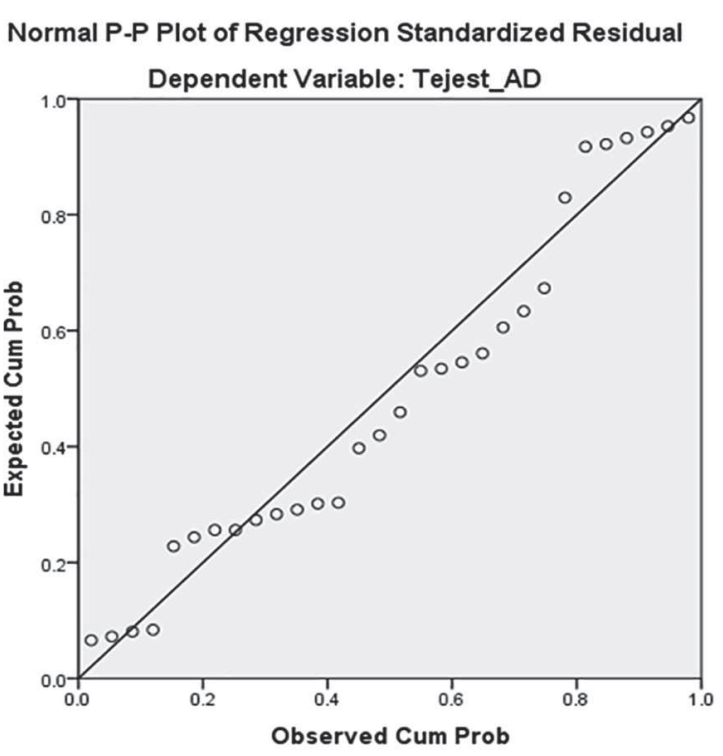

Fig. 3. Directly proportional dependence of the correlation between the SSR latency and the AD severity

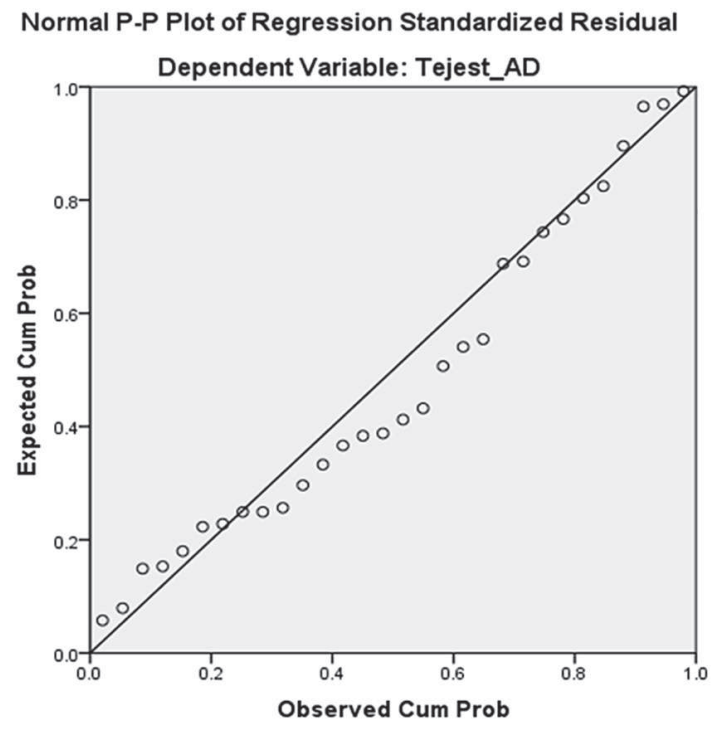

Fig. 4. Directly proportional dependence of the correlation between the SSR amplitude and the AD severity

The performed analysis showed the differences in the median latency values by patient groups, with mild and severe dysfunctions. In regard to upper limb latency times in patients with mild autonomic dysfunctions, the longest latency time was observed in patients with ICS in LMCA with IC (1.73 s). In the group of severe $\mathrm{AD}$, the longest latency time was in patients with ICS in RMCA with IC (2.28 s). As for lower limb latency times, in the group of individuals with mild autonomic dysfunctions, the longest dis- 
tal latency time was observed in patients with ICS in RMCA with IC ( $2.3 \mathrm{~s}$ ) and in the group of patients

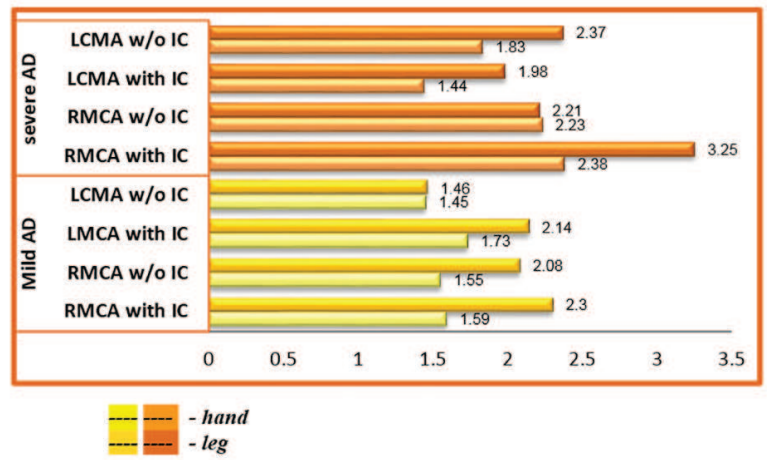

Fig. 5. Distribution of the median values of SSR latencies in patients with mild and severe $A D$

with severe $\mathrm{AD}$ - in patients with ICS in RMCA with IC ( 3.25 s) (Fig. 5).

In regard to the SSR amplitude values, the lowest one for upper limb was observed in patients with ICS in RMCA without IC $(1.36 \mathrm{mV})$ with mild autonomic dysfunctions and in patients with ICS in LMCA without IC $(0.56 \mathrm{mV})$ with severe AD. The lowest lower limb values were observed in patients with ICS in LMCA without IC $(1.18 \mathrm{mV})$ with mild autonomic dysfunctions and in patients with ICS in

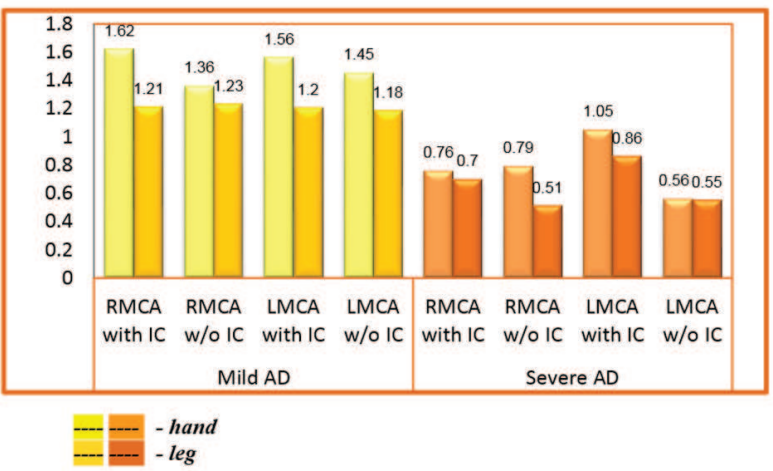

Fig. 6. Distribution of the median values of SSR amplitudes in patients with mild and severe $A D$

RMCA without IC ( $0.51 \mathrm{mV})$ with severe autonomic dysfunction (Fig. 6).

We have established a statistically significant difference between the group of patients with mild and severe autonomic dysfunctions for the four variables: hand latency, leg latency, hand amplitude, and leg amplitude $(\mathrm{p}<0.05)$ (Table. 2$)$.
Table 2. Comparative analysis of the SSR parameters in patients with mild and severe autonomic dysfunctions

\begin{tabular}{|c|c|c|c|}
\hline \multirow[t]{2}{*}{ Comparisons } & \multicolumn{2}{|c|}{$\begin{array}{l}\text { Confidence } \\
\text { interval 95\% }\end{array}$} & \multirow[t]{2}{*}{$\mathrm{p}$} \\
\hline & Hand & Leg & \\
\hline $\begin{array}{l}\text { Hand latency } \\
\text { (mild with severe } \\
\text { dysfunction) }\end{array}$ & -0.11 & -0.84 & 0.004 \\
\hline $\begin{array}{l}\text { Leg latency } \\
\text { (mild with severe } \\
\text { dysfunction) }\end{array}$ & -0.13 & -1.18 & 0.007 \\
\hline $\begin{array}{l}\text { Hand amplitude } \\
\text { (mild with severe } \\
\text { dysfunction) }\end{array}$ & 0.99 & 0.36 & $<0.0001$ \\
\hline $\begin{array}{l}\text { Leg amplitude } \\
\text { (mild with severe } \\
\text { dysfunction) }\end{array}$ & 0.85 & 0.25 & 0.001 \\
\hline
\end{tabular}

From the analysis we have performed on the SSR result, we concluded that there were statistically significant differences in regard to the latency times and the amplitudes between the controls and the patients with stroke, which is also confirmed by a review of the literature $(1,9,10,11)$. The bilaterally prolonged latencies and the decreased SSR amplitudes as a consequence of a unilateral ischemic lesion in our patients, can be explained with the impact of the complex bilateral associations of the medullar reticular formation with the cerebral cortex.

When comparing the obtained results from patients with left hemispheric and those with right hemispheric strokes, we have concluded that there are longer latency times and lower amplitudes in patients with right hemispheric strokes with the most marked abnormal values being in patients with ICS in RMCA with IC. This data confirms the prevailing importance of the right insular cortex in the autonomic sympathetic regulation (10).

\section{CONCLUSION}

The data from our research shows that the patients with severe autonomic dysfunction have a significantly more marked disturbances in the SSR parameters compared to the deviations in patients with mild autonomic dysfunctions. The established correlation between the SSR parameters and the severity of the cardiovascular autonomic dysfunction is most likely related to the degree of sympathetic adrenal axis disorder in patients with acute ischemic stroke. 


\section{REFERENCES}

1. Korpelainen JT, Tolonen U, Sotaniemi KA, Myllyla VV. Suppressed sympathetic skin response in brain infarction. Stroke,1993;24:1389-92

2. Vetrugno R, Liguari R, Cortelli P, Montagna P, Sympathetic skin response: basic mechanisms and clinical applications. Clin Auton Res 2003, $13: 256-270$

3. Chroni E, Andreas Argyriou A., Polychronopoulos P, Sirrou V. The effect of stimulation technique on sympathetic skin responses in healthy subjects Clin Auton Res 2006, 16:396-400

4. Claus D, Schondorf R. Sympathetic skin response. The International Federation of Clinical Neurophysiology. Electroencephalogr Clin Neurophysiol Suppl. 1999;52:277-82.

5. Muslumanoglu L, Aki S, Turkdogan D, Us O, Akyuz G. Involvement of sympathetic reflex activity in patients with acute and chronic stroke: A comparison with functional motor capacity. Arch Phys Med Rehabil. 2004 Mar;85(3):470-3.

6. Kobayashi R, Koike Y, Hirayama M, Ito H, Sobue G. Skin sympathetic nerve function during sleep--a study with effector responses.Auton Neurosci. 2003 Jan 31;103(1-2):121-6.

7. Muslumanoglu L, Akyuz G, Aki S, Karsidag S. Evaluation of autonomic nervous system functions in post-stroke patients. Am J Phys Med Rehabil, 2002, 81:721-725.

8. Łabuz-Roszak B, Pierzchała K. Stroke induces disturbances of autonomic system function Neurol Neurochir Pol. 2007 Nov-Dec;41(6):495-503..

9. Erciyas H, Topalkara K, Topaktaş S, Akyüz G. Suppression of cardiac parasympathetic functions in patients with right hemispheric stroke. Eur J Neurol 1999, 6:685-690

10. Selçuk B, Ersoz M, Inanir M, Kurtaran A, Akyuz M. Sympathetic skin responses in hemiplegic patients with and without complex regional pain syndrome. Neurol India. 2006 Sep; 54(3):279-82.

11. Cakır T, Evcik FD, Subaşı V, Demirdal US, Kavun$\mathrm{cu} \mathrm{V}$. Investigation of the $\mathrm{H}$ reflexes, $\mathrm{F}$ waves and sympathetic skin response with electromyography (EMG) in patients with stroke and the determination of the relationship with functional capacity. Acta Neurol Belg. 2014 Dec 7. 